\title{
THE PERTURBATION THEORY FOR THE DRAZIN INVERSE AND ITS APPLICATIONS II
}

\author{
VLADIMIR RAKOČEVIĆ and YIMIN WEI
}

(Received 3 November 1999; revised 26 July 2000)

Communicated by K. Ecker

\begin{abstract}
We study the perturbation of the generalized Drazin inverse for the elements of Banach algebras and bounded linear operators on Banach space. This work, among other things, extends the results obtained by the second author and Guorong Wang on the Drazin inverse for matrices.
\end{abstract}

2000 Mathematics subject classification: primary 47A05, 47A53, 15 A09.

Keywords and phrases: generalized Drazin inverse, Banach algebra, perturbation, linear equation.

\section{Introduction and preliminaries}

Let us point out that the important applications of perturbation of the Drazin inverse are to, for example, singular perturbations of autonomous linear systems of differential equations and perturbation of continuous semigroups of bounded linear operators (see [2-5]). The perturbation properties of the Drazin inverse for matrices were investigated by Yimin Wei [10] and Yimin Wei and Guorong Wang [11]. In the present paper we study the perturbation of the generalized Drazin inverse introduced recently by Koliha [7] (see also Koliha and Rakočević [8]). We start in the Banach algebra setting, and then move to bounded linear operators.

We denote by $\mathscr{A}$ a complex Banach algebra with identity 1 . For an element $a \in \mathscr{A}$ we denote by $\sigma(a)$ the spectrum of $a$. We write acc $\sigma(a)$ for the set of all accumulation points of $\sigma(a)$. By qNil $(\mathscr{A})$ we denote the set of all quasinilpotent element of $\mathscr{A}$.

The work of the first author was supported by the the Sci. Found of Serbia, grant number 04M03, through Matematički Institut.

The second author is supported by: Project 19901006 by National Natural Science Foundation of China, Doctoral Point Foundation of China, Youth Science Foundation of Universities in Shanghai of China and Laboratory of Computational Physics in Beijing of China.

(C) 2001 Australian Mathematical Society 0263-6115/2001 \$A2.00+0.00 
DEFINITION 1.1. Let $a \in \mathscr{A}$. Following [7], we say that $a$ is Drazin invertible if there exists $b \in \mathscr{A}$ such that

$$
a b=b a, \quad a b^{2}=b, \quad a^{2} b-a \in \mathrm{qNil}(\mathscr{A}) .
$$

Such $b$, if it exists, is unique [7], it is called the generalized Drazin inverse of $a$, and is denoted by $a^{D}$. If $a^{2} b-a$ is in fact nilpotent, then $a^{D}$ is the conventional Drazin inverse of $a$ (see $[6,7])$. The Drazin index $i(a)$ of $a$ is equal to $k$ if $a^{2} b-a$ is nilpotent of index $k$, otherwise $i(a)=\infty$. If $i(a)=1$, then $a^{D}$ is denoted by $a^{\#}$ and is called the group inverse of $a$. From this point on we use the term 'Drazin inverse' instead of 'generalized Drazin inverse' (see also [8]). Let us recall [7, Theorem 4.2] that $a$ has a Drazin inverse if and only if $0 \notin \operatorname{acc} \sigma(a)$.

With each Drazin invertible element $a$ we associate the core part of $a$ defined by $a^{2} a^{D}$ and the quasinilpotent part of $a$ defined by $a^{2} a^{D}-a$. Now we have the following core-quasinilpotent decomposition of $a$

$$
a=\left(a^{2} a^{D}\right)+\left(a-a^{2} a^{D}\right) .
$$

For the applications of the core-quasinilpotent decomposition see $([7,8])$.

Let $a \in \mathscr{A}$ be Drazin invertible. Following [11], we say that $b \in \mathscr{A}$ obeys the condition $(\mathscr{W})$ at $a$ if

$$
b-a=a a^{D}(b-a) a a^{D} \text { and }\left\|a^{D}(b-a)\right\|<1 .
$$

Let us remark that the condition

$$
b-a=a a^{D}(b-a) a a^{D}
$$

is equivalent to the condition

$$
b-a=a a^{D}(b-a)=(b-a) a a^{D} .
$$

Basic auxiliary results are summarized in the following lemma (see also [11, Theorem 3.1 and Theorem 3.2]). For the sake of completeness we include a proof.

LEMMA 1.1. Let $a \in \mathscr{A}$ be Drazin invertible, and let $b \in \mathscr{A}$ obey the condition (W) at a. Then

(i) $b=a\left(1+a^{D}(b-a)\right)$;

(ii) $b=\left(1+(b-a) a^{D}\right) a$;

(iii) $1+a^{D}(b-a)$ and $1+(b-a) a^{D}$ are invertible, and

$$
\left(1+a^{D}(b-a)\right)^{-1} a^{D}=a^{D}\left(1+(b-a) a^{D}\right)^{-1} .
$$


PROOF. To prove (i) and (ii) let us remark that by (1.4) we have

$$
b=a+(b-a)=a+a a^{D}(b-a)=a\left(1+a^{D}(b-a)\right)
$$

and

$$
b=a+(b-a)=a+(b-a) a^{D} a=\left(1+(b-a) a^{D}\right) a .
$$

Clearly, the condition $\left\|a^{D}(b-a)\right\|<1$ implies that $1+a^{D}(b-a)$ and $1+(b-a) a^{D}$ are invertible. Finally, (1.5) follows by direct verification.

\section{The perturbation results for the Drazin inverse in Banach algebras}

Now we prove the main results of this section.

THEOREM 2.1. Let $a \in \mathscr{A}$ be Drazin invertible, and let $b \in \mathscr{A}$ obey the condition $(\mathscr{W})$ at $a$. Then $b$ is Drazin invertible, $b b^{D}=a a^{D}, b^{D}=\left(1+a^{D}(b-a)\right)^{-1} a^{D}=$ $a^{D}\left(1+(b-a) a^{D}\right)^{-1}$ and $i(a)=i(b)$.

PROOF. By Lemma 1.2 (iii) we know that $1+a^{D}(b-a)$ and $1+(b-a) a^{D}$ are invertible and

$$
\left(1+a^{D}(b-a)\right)^{-1} a^{D}=a^{D}\left(1+(b-a) a^{D}\right)^{-1} .
$$

Set $\tilde{b}=\left(1+a^{D}(b-a)\right)^{-1} a^{D}=a^{D}\left(1+(b-a) a^{D}\right)^{-1}$. We prove that $b$ is Drazin invertible and that $b^{D}=\tilde{b}$. First we prove that $b$ and $\tilde{b}$ are commuting. By Lemma 1.2 (i) we have

$$
b \tilde{b}=a\left(1+a^{D}(b-a)\right)\left(1+a^{D}(b-a)\right)^{-1} a^{D}=a a^{D}
$$

and by Lemma 1.2 (ii) we get

$$
\tilde{b} b=a^{D}\left(1+(b-a) a^{D}\right)^{-1}\left(1+(b-a) a^{D}\right) a=a^{D} a .
$$

Hence, by (2.1) and (2.2) we get

$$
b \tilde{b}=\tilde{b} b
$$

Therefore

$$
\tilde{b}-b \tilde{b}^{2}=\tilde{b}(1-b \tilde{b})=\left(1+a^{D}(b-a)\right)^{-1} a^{D}\left(1-a^{D} a\right)=0,
$$

and $b \tilde{b}^{2}=\tilde{b}$. Finally, using (2.1) and (1.4), we get

$$
\begin{aligned}
b-b^{2} \tilde{b} & =b(1-b \tilde{b})=b\left(1-a a^{D}\right) \\
& =a\left(1-a a^{D}\right)+(b-a)\left(1-a a^{D}\right)=a-a^{2} a^{D}
\end{aligned}
$$


which is quasinilpotent. We conclude that $b$ is Drazin invertible with $b^{D}=\tilde{b}$. Equations (2.1) and (2.2) show that $b b^{D}=a a^{D}$. From (2.4) we conclude that $i(a)=i(b)$.

Let us remark that as a direct corollary of Theorem 2.1 we obtain the known result for matrices [11, Theorem 1]. The next corollary is a generalization of [11, Theorem 3.2].

COROLlaRY 2.2. Let $a \in \mathscr{A}$ be Drazin invertible and let $b \in \mathscr{A}$ obey the condition $(\mathscr{W})$ at $a$. Then $b$ is Drazin invertible, and we have

$$
\frac{\left\|b^{D}-a^{D}\right\|}{\left\|a^{D}\right\|} \leq \frac{\left\|a^{D}(b-a)\right\|}{1-\left\|a^{D}(b-a)\right\|} .
$$

ProOF. By Theorem 2.1 we have

$$
\begin{aligned}
b^{D}-a^{D} & =\left(1+a^{D}(b-a)\right)^{-1} a^{D}-a^{D}=\left[\left(1+a^{D}(b-a)\right)^{-1}-1\right] a^{D} \\
& =\left(1+a^{D}(b-a)\right)^{-1}\left(a^{D}(b-a)\right) a^{D} .
\end{aligned}
$$

Hence

$$
\begin{aligned}
\left\|b^{D}-a^{D}\right\| & \leq\left\|\left(1+a^{D}(b-a)\right)^{-1}\right\|\left\|\left(a^{D}(b-a)\right)\right\|\left\|a^{D}\right\| \\
& \leq \frac{\left\|a^{D}(b-a)\right\|}{1-\left\|a^{D}(b-a)\right\|}\left\|a^{D}\right\|,
\end{aligned}
$$

and we get (2.5).

COROLlaRY 2.3. Let $a \in \mathscr{A}$ be Drazin invertible, and let $b \in \mathscr{A}$ obey the condition $(\mathscr{W})$ at $a$. Then $b$ is Drazin invertible, and we have

$$
\frac{\left\|a^{D}\right\|}{1+\left\|a^{D}(b-a)\right\|} \leq\left\|b^{D}\right\| \leq \frac{\left\|a^{D}\right\|}{1-\left\|a^{D}(b-a)\right\|} .
$$

ProOF. By Theorem 2.1 we know that

$$
b^{D}=\left(1+a^{D}(b-a)\right)^{-1} a^{D},
$$

hence

$$
a^{D}=\left(1+a^{D}(b-a)\right) b^{D} .
$$

Now

$$
\begin{aligned}
& \left\|b^{D}\right\| \leq\left\|\left(1+a^{D}(b-a)\right)^{-1}\right\|\left\|a^{D}\right\| \leq \frac{\left\|a^{D}\right\|}{1-\left\|a^{D}(b-a)\right\|}, \\
& \left\|a^{D}\right\| \leq\left\|\left(1+a^{D}(b-a)\right)\right\|\left\|b^{D}\right\| \leq\left(1+\left\|a^{D}(b-a)\right\|\right)\left\|b^{D}\right\|,
\end{aligned}
$$

and we get (2.8). 
COROLlaRY 2.4. Let $a \in \mathscr{A}$ be Drazin invertible, $b \in \mathscr{A}$ obey the condition (W) at $a$, and $\left\|a^{D}(b-a)\right\|<1 / 2$. Then $b$ is Drazin invertible, and a obeys the condition (W) at $b$.

PROOF. By Theorem 2.1 we know that $b b^{D}=a a^{D}$. Hence $a-b=b b^{D}(a-b) b b^{D}$. Again, by Theorem 2.1, we have

$$
\begin{aligned}
\left\|b^{D}(a-b)\right\| & =\left\|\left(1+a^{D}(b-a)\right)^{-1} a^{D}(a-b)\right\| \\
& \leq\left\|\left(1+a^{D}(b-a)\right)^{-1}\right\|\left\|a^{D}(a-b)\right\| \leq \frac{\left\|a^{D}(a-b)\right\|}{1-\left\|a^{D}(b-a)\right\|}<1 .
\end{aligned}
$$

This completes the proof.

Let us remark that the next corollary can be obtained as in [11, Corollary 3.2] and we omit the proof.

COROllaRY 2.5. Let $a \in \mathscr{A}$ be Drazin invertible, let $b \in \mathscr{A}$ obey the condition $(\mathscr{W})$ at $a$ and let $\left\|a^{D}\right\|\|b-a\|<1$. Then $b$ is Drazin invertible and we have

$$
\frac{\left\|b^{D}-a^{D}\right\|}{\left\|a^{D}\right\|} \leq \frac{k_{D}(a)\|b-a\| /\|a\|}{1-k_{D}(a)\|(b-a)\| /\|a\|},
$$

where

$$
k_{D}(a)=\|a\|\left\|a^{D}\right\|
$$

is defined as the condition number with respect to the Drazin inverse.

We finish this section with some algebraic properties of Drazin inverse. Let us remark that it is well known that if $a, b \in \mathscr{A}$ are Drazin invertible, then $a b$ is not necessary Drazin invertible, and if $a b$ is Drazin invertible, then in general $(a b)^{D} \neq$ $b^{D} a^{D}$. In the next propositions we show that if $b$ obeys the condition $(\mathscr{W})$ at $a$ we can be more precise.

PROPOSITION 2.6. Let $a \in \mathscr{A}$ be Drazin invertible and let $b \in \mathscr{A}$ obey the condition (W) at a. Then:

(i) $a b^{D}$ has group inverse and $\left(a b^{D}\right)^{\#}=b a^{D}$.

(ii) $b^{D} a$ has group inverse and $\left(b^{D} a\right)^{\#}=a^{D} b$.

(iii) $b a^{D}$ has group inverse and $\left(b a^{D}\right)^{\#}=a b^{D}$.

(iv) $a^{D} b$ has group inverse and $\left(a^{D} b\right)^{\#}=b^{D} a$.

PROOF. It is enough to prove (i) and (ii). To prove (i) let us remark that by Theorem 2.1 we have $b b^{D}=a a^{D}$. Hence

$$
\left(a b^{D}\right)\left(b a^{D}\right)=a\left(b^{D} b\right) a^{D}=a\left(a^{D} a\right) a^{D}=a a^{D}
$$


and

$$
\left(b a^{D}\right)\left(a b^{D}\right)=b\left(a^{D} a\right) b^{D}=b\left(b^{D} b\right) b^{D}=b b^{D}=a a^{D} .
$$

Thus we prove that

$$
\left(a b^{D}\right)\left(b a^{D}\right)=\left(b a^{D}\right)\left(a b^{D}\right) .
$$

Further,

$$
\begin{aligned}
\left(a b^{D}\right)\left(b a^{D}\right)\left(a b^{D}\right) & =a a^{D}\left(a b^{D}\right)=a\left(a^{D} a\right) b^{D} \\
& =a\left(b^{D} b\right) b^{D}=a\left(b^{D} b b^{D}\right)=a b^{D} .
\end{aligned}
$$

Finally,

$$
\left(b a^{D}\right)\left(a b^{D}\right)\left(b a^{D}\right)=\left(b a^{D}\right) a a^{D}=b\left(a^{D} a a^{D}\right)=b a^{D} .
$$

Now by (2.15), (2.16) and (2.17) we get (i). Let us remark that (ii) can be proved similarly, and we omit the proof.

PROPOSITION 2.7. Let $a \in \mathscr{A}$ be Drazin invertible and let $b_{1}, b_{2} \in \mathscr{A}$ obey the condition (W) at a. Then $b_{1} b_{2}$ is Drazin invertible and $\left(b_{1} b_{2}\right)^{D}=b_{2}^{D} b_{1}^{D}$.

PROOF. The elements $b_{1}, b_{2}$ are Drazin invertible and $b_{i} b_{i}^{D}=a a^{D}$ by Theorem 2.1. Hence

$$
b_{i} a a^{D}=a a^{D} b_{i} \quad \text { and } \quad b_{i}^{D} a a^{D}=a a^{D} b_{i}^{D}=b_{i}^{D}, \quad i=1,2 .
$$

Write $c=b_{2}^{D} b_{1}^{D}$. We show that $c$ is the Drazin inverse of $b_{1} b_{2}$. First,

$$
\left(b_{1} b_{2}\right) c=b_{1}\left(b_{2} b_{2}^{D}\right) b_{1}^{D}=b_{1}\left(a a^{D}\right) b_{1}^{D}=\left(a a^{D}\right)\left(b_{1} b_{1}^{D}\right)=\left(a a^{D}\right)^{2}=a a^{D} .
$$

Similarly

$$
c\left(b_{1} b_{2}\right)=a a^{D}=\left(b_{1} b_{2}\right) c
$$

Further, by (2.18)

$$
c^{2}\left(b_{1} b_{2}\right)=c\left(c b_{1} b_{2}\right)=c a a^{D}=b_{2}^{D}\left(b_{1}^{D} a a^{D}\right)=b_{2}^{D} b_{1}^{D}=c .
$$

Finally, we observe that in view of Lemma 1.2,

$$
\begin{aligned}
b_{i}\left(1-a^{D} a\right) & =\left(a+\left(b_{i}-a\right) a^{D} a\right)\left(1-a^{D} a\right) \\
& =a\left(1-a^{D} a\right)=\left(1-a^{D} a\right) a, \quad i=1,2 .
\end{aligned}
$$

Hence,

$$
\begin{aligned}
b_{1} b_{2}-\left(b_{1} b_{2}\right)^{2} c & =b_{1} b_{2}\left(1-b_{1} b_{2} c\right)=b_{1} b_{2}\left(1-a^{D} a\right) \\
& =b_{1}\left(1-a^{D} a\right) a=a\left(1-a^{D} a\right) a,
\end{aligned}
$$

where $a\left(1-a^{D} a\right) a=a\left(a-a^{D} a^{2}\right)=\left(a-a^{D} a^{2}\right) a$ is quasinilpotent. This show that $\left(b_{1} b_{2}\right)^{D}=c$. 
As a corollary we have the following result.

COROLlaRY 2.8. Let $a \in \mathscr{A}$ be Drazin invertible and let $b \in \mathscr{A}$ obey the condition (W) at $a$. Then $a b$ and ba are Drazin invertible, $(a b)^{D}=b^{D} a^{D}$ and $(b a)^{D}=a^{D} b^{D}$.

PROOF. The result follows from Proposition 2.7.

\section{Applications}

In this section we give some applications of Theorem 2.1, Corollary 2.2 and Corollary 2.3. The results in this paper extend the results obtained by Yimin Wei and Guorong Wang [11, Applications].

Let $X$ be an infinite-dimensional complex Banach space and denote the set of bounded linear operators on $X$ by $B(X)$. Throughout this paper, $N(T)$ and $R(T)$ denote the null space and the range space of $T \in B(X)$, respectively.

Let us recall that the following result was obtained by Koliha [7], and it is a generalization of Lay's result [9] for the finite index Drazin inverse.

THEOREM 3.1. Let $A \in B(X)$ be such that $0 \notin \operatorname{acc} \sigma(A)$. If $A^{D}$ is the Drazin inverse of $A$ as an element of the algebra $B(X)$, then $X=R\left(A^{D} A\right) \oplus N\left(A^{D} A\right)$, $A=A_{1} \oplus A_{2}$ with respect to this direct sum and $A^{D}=A_{1}^{-1} \oplus O$.

We shall consider error bounds for the Drazin inverse in $B(X)$. Let us mention that some related results were obtained for the Drazin inverse of matrix [11] and for the Moore-Penrose inverse of matrix [1].

Let us consider the equation

$$
A x=b,
$$

where $A$ is Drazin invertible. We study the sensitivity of the solution $x$ to variation in the data $b$ and $A$, provided that $b$ and $x$ are in $R\left(A^{D}\right)$.

THEOREM 3.2. Let $A \in B(X)$ be Drazin invertible and let $b, c \in R\left(A^{D}\right)$. If $x, y \in R\left(A^{D}\right)$ satisfy $A x=b$ and $A y=c$, then

$$
\frac{\|y-x\|}{\|x\|} \leq k_{D}(A) \frac{\|c-b\|}{\|b\|} \text {. }
$$

PROOF. Since $A^{D} A$ is the (oblique) projection onto $R\left(A^{D}\right)$, from $x, y, b, c \in$ $R\left(A^{D}\right), A x=b$ and $A y=c$, we have $x=A^{D} A x=A^{D} b$ and $y=A^{D} A y=A^{D} c$. Hence,

$$
\|y-x\| \leq\left\|A^{D}\right\|\|c-b\|
$$


and

$$
\|b\| \leq\|A\|\|x\| .
$$

Clearly, (3.1) follows from (3.2) and (3.3).

THEOREM 3.3. Let $A \in B(X)$ be Drazin invertible, let $B \in B(X)$ obey the condition $(\mathscr{W})$ at $A$, and let $b \in R\left(A^{D}\right)$. If $x, y \in R\left(A^{D}\right)$ satisfy $A x=b$ and $B y=b$, then

$$
\frac{\|y-x\|}{\|x\|} \leq k_{D}(A) \frac{\left\|A^{D}(B-A)\right\|}{1-\left\|A^{D}(B-A)\right\|} .
$$

PROOF. By Theorem 2.1 we know that $A^{D} A=B^{D} B$, and $R\left(A^{D}\right)=R\left(B^{D}\right)$. Hence, $x=A^{D} A x=A^{D} b$ and $y=B^{D} B y=B^{D} b$. Further,

$$
\|y-x\| \leq\left\|B^{D}-A^{D}\right\|\|b\| \leq\left\|B^{D}-A^{D}\right\|\|A\|\|x\| .
$$

Finally, (3.4) follows by Corollary 2.2 and (3.5).

THEOREM 3.4. Let $A \in B(X)$ be Drazin invertible, let $B \in B(X)$ obey the condition (W) at $A$, and let $b, c \in R\left(A^{D}\right)$. If $x, y \in R\left(A^{D}\right)$ satisfy $A x=b$ and $B y=c$, then

$$
\|y-x\| \leq \frac{\left\|A^{D}\right\|}{1-\left\|A^{D}(B-A)\right\|}\left(\left\|A^{D}(B-A)\right\|\|b\|+\|c-b\|\right) .
$$

Proof. By Theorem 2.1 we know that $A^{D} A=B^{D} B$, and $R\left(A^{D}\right)=R\left(B^{D}\right)$. Hence, $x=A^{D} A x=A^{D} b$ and $y=B^{D} B y=B^{D} c$. Further,

$$
y-x=B^{D} c-A^{D} b=\left(B^{D}-A^{D}\right) b+B^{D}(c-b)
$$

and we obtain

$$
\|y-x\| \leq\left\|B^{D}-A^{D}\right\|\|b\|+\left\|B^{D}\right\|\|c-b\| .
$$

Clearly, (3.6) follows by Corollary 2.2, Corollary 2.3 and (3.7).

Let us remark that if it is assumed in addition to all the other hypotheses that $\left\|A^{D}\right\|\|B-A\|<1$, then the expression $\left\|A^{D}(B-A)\right\|$ in Theorems 3.3-3.4 can be replaced by $\left\|A^{D}\right\|\|B-A\|$.

Now, as a corollary, we get a generalization of [11, Theorem 4.1]. Our formulation of that result is somewhat different from that of Yimin Wei and Guorong Wang's, but consistent with Theorem 3.4. 
THEOREM 3.5. Let the hypotheses of Theorem 3.4 be satisfied together with the inequality $\left\|A^{D}\right\|\|B-A\|<1$. Then

$$
\frac{\|y-x\|}{\|x\|} \leq \frac{k_{D}(A)}{1-k_{D}(A)\|B-A\| /\|A\|}\left(k_{D}(A) \frac{\|B-A\|}{\|A\|}+\frac{\|c-b\|}{\|b\|}\right) .
$$

PROOF. The proof follows by Theorem 3.4 and remark that $\|b\| \leq\|A\|\|x\|$.

Let us mention that Theorem 3.5, unlike Theorem 3.4, deals with relative errors.

\section{Acknowledgement}

We wish to thank the referee for his valuable comments and suggestions.

\section{References}

[1] A. Ben-Israel, 'On error bounds for generalized inverses', SIAM J. Numer. Anal. 3 (1966), 585-592.

[2] S. L. Campbell, 'Singular perturbation of autonomous linear systems II', J. Differential Equations 29 (1978), 362-373.

[3] - Singular systems of differential equations I, Res. Notes Math. 40 (Pitman, London, 1980).

[4] - Singular systems of differential equations II, Res. Notes Math. 61 (Pitman, London, 1982).

[5] S. L. Campbell and N. J. Rose, 'Singular perturbation of autonomous linear systems', SIAM J. Math. Anal. 10 (1979), 542-551.

[6] M.P. Drazin, 'Pseudoinverse in associative rings and semigroups', Amer. Math. Monthly 65 (1958), 506-514.

[7] J. J. Koliha, 'A generalized Drazin inverse', Glasgow Math. J. 38 (1996), 367-381.

[8] J. J. Koliha and V. Rakočević, 'Continuity of the Drazin inverse II', Studia Math. 131 (1998), 167-177.

[9] D. C. Lay, 'Spectral properties of generalized inverses of linear operators', SIAM J. Appl. Math. 29 (1975), 103-109.

[10] Yimin Wei, 'On the perturbation of the group inverse and oblique projection', Appl. Math. Comput. 98 (1998), 29-42.

[11] Yimin Wei and Wang Guorohg Wang, 'The perturbation theory for the Drazin inverse and its applications', Linear Algebra Appl. 258 (1997), 179-186.

University of Niš

Faculty of Philosophy

Department of Mathematics

Ćirila and Metodija 2

18000 Niš

Yugoslavia-Serbia

e-mail: vrakoc@bankerinter.net
Department of Mathematics and Laboratory of Nonlinear Science

Fudan University

Shanghai 200433

P. R. China

e-mail: ymwei@fudan.edu.cn 
